\title{
Partner Selection of Strategic Alliance in Shipping Enterprises
}

\author{
Xiao-Xue REN ${ }^{1, a^{*}}$, Xin-Ran WANG ${ }^{1, b}$, Ru ZHANG $^{1, c}$, Kai DING ${ }^{2, \mathrm{~d}}$ \\ ${ }^{1}$ Shandong Jiaotong University, Ji'nan, 250357, China \\ ${ }^{2}$ Dalian Maritime University, Dalian, 116026, China \\ a1587351294@qq.com, b1193015465@qq.com, '649953580@qq.com, ${ }^{\mathrm{d}} 787667959 @ q q . c o m$ \\ *Xiao-Xue REN
}

Keywords: Shipping enterprise, AHP, Strategic alliance.

\begin{abstract}
The Analytic Hierarchy Process (AHP) was chosen to solve partner issue of shipping enterprise strategic alliance in this paper, taking China Ocean Container Transportation Co. Ltd (COSCO Co., Ltd). As an example, firstly partner selection evaluation index were discussed, and then the structure diagram of strategic alliance partner selection and evaluation of the various elements of the design criterion layers was illustrated, and the weight of each index was calculated respectively, finally how to choose the best partner according to the weight was settled.
\end{abstract}

\section{Introduction}

COSCO Co., Ltd is a transnational enterprise group which operates shipping, logistics and other related business. It is now faced with the enormous competitive pressure from other international alliances of large transport companies [1]. Therefore the group decided to cooperate with several friendly logistics companies to form the vertical strategic alliance. So the analytic hierarchy process was used to confirm the weights of the 4 companies.

\section{AHP Method is Used to Select Shipping Enterprise Strategic Partner}

\section{Determine the Partner Selection Evaluation Index}

The selection evaluation index is shown in Table 1.

Table 1 Strategic partner selection evaluation index

\begin{tabular}{|c|c|c|}
\hline \multirow{14}{*}{$\begin{array}{l}\text { The strategic partner selection } \\
\text { evaluation index of shipping } \\
\text { enterprises }\end{array}$} & \multirow{6}{*}{ Compatibility } & Compatibility of strategic ideas \\
\hline & & Organization management structure \\
\hline & & Value creation potential \\
\hline & & Benefit and risk sharing \\
\hline & & Human resources \\
\hline & & Historical cooperation \\
\hline & \multirow{4}{*}{ Ability } & Operation ability \\
\hline & & Emergency response capability \\
\hline & & Internal coordination ability \\
\hline & & Core business \\
\hline & \multirow{4}{*}{ Input } & Asset scale \\
\hline & & Advanced transport equipment \\
\hline & & Information level \\
\hline & & personnel \\
\hline
\end{tabular}


If there were 4 shipping enterprises for the selection as objects, it could be seen as 4 options, the structure of the strategic alliance partner selection through the AHP hierarchy is shown through Fig. 1.

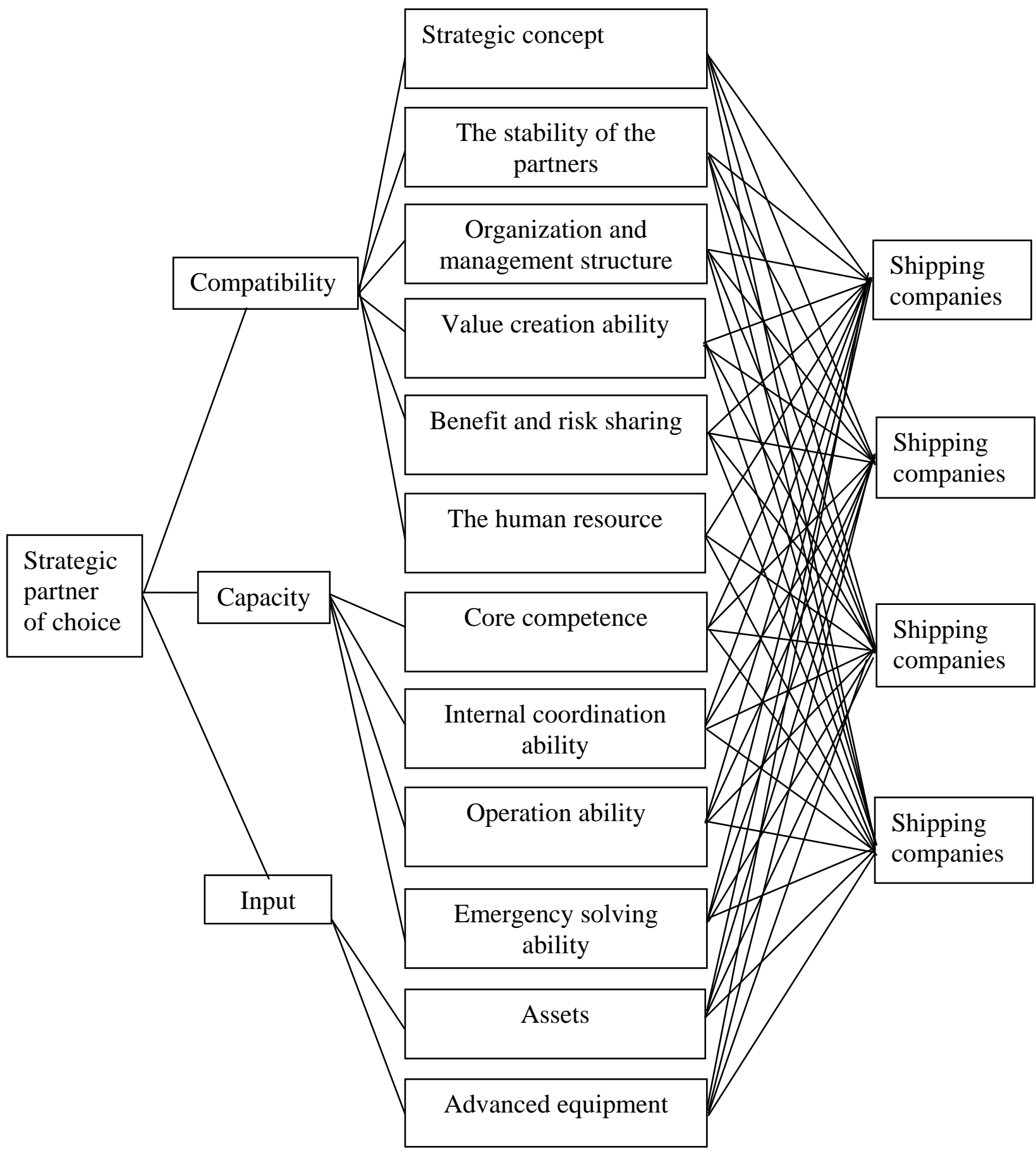

Fig. 1 Choice of enterprise hierarchy

One expert group of the design criteria for the evaluation of the elements was organized to evaluate every factor. Then get the results, it is shown in Table 2. 
Table 2 Evaluation criteria layers table of the elements

\begin{tabular}{|l|l|l|l|}
\hline & Compatibility & Ability & Input \\
\hline Compatibility & 1 & 3 & 5 \\
\hline Ability & $1 / 3$ & 1 & 3 \\
\hline Input & $1 / 5$ & $1 / 3$ & 1 \\
\hline Total & $23 / 15$ & $13 / 3$ & 9 \\
\hline
\end{tabular}

After normalizing the judgment matrix of the criterion layer, the weights of each criterion were obtained, as shown in Table 3.

Table 3 The normalized weight

\begin{tabular}{|l|l|l|l|l|}
\hline & Compatibility & Ability & Input & Weight \\
\hline Compatibility & $15 / 23$ & $9 / 13$ & $5 / 9$ & 0.663 \\
\hline Ability & $5 / 23$ & $3 / 13$ & $3 / 9$ & 0.261 \\
\hline Input & $3 / 23$ & $1 / 13$ & $1 / 9$ & 0.106 \\
\hline Total & & & & 1.000 \\
\hline
\end{tabular}

$$
A W=\left(\begin{array}{ccc}
1 & 3 & 5 \\
1 / 3 & 1 & 3 \\
1 / 5 & 1 / 3 & 1
\end{array}\right) \cdot\left(\begin{array}{l}
0.663 \\
0.261 \\
0.106
\end{array}\right)=\left(\begin{array}{c}
1.946 \\
0.790 \\
0.320
\end{array}\right)
$$

$$
\lambda=(1.946 / 0.633+0.790 / 0.261+0.320 / 0.106) / 3=3.04, R I=0.52,
$$

$C I=(\lambda-n) /(n-1)=0.03, C R=C I / R I=0.038<0.1$, complying with the consistency check.

The weights of each criterion layer were determined and then check the consistency. The weight of each sub criterion layer under the principle of computational compatibility was listed [2].The primary data were shown in Table 4 and the normalized data is shown in Table 5.

Table 4 Evaluation of the sub criterion under the compatibility guideline

\begin{tabular}{|l|l|l|l|l|l|l|}
\hline & $\begin{array}{l}\text { Strategic } \\
\text { concept }\end{array}$ & $\begin{array}{l}\text { Partner } \\
\text { stability }\end{array}$ & $\begin{array}{l}\text { Organization } \\
\text { management } \\
\text { structure }\end{array}$ & $\begin{array}{l}\text { Value } \\
\text { creation } \\
\text { ability }\end{array}$ & $\begin{array}{l}\text { Benefit and } \\
\text { risk sharing }\end{array}$ & $\begin{array}{l}\text { Human } \\
\text { resources }\end{array}$ \\
\hline $\begin{array}{l}\text { Strategic } \\
\text { concept }\end{array}$ & 1 & $1 / 3$ & $1 / 2$ & $1 / 4$ & $1 / 5$ & $1 / 4$ \\
\hline $\begin{array}{l}\text { Partner } \\
\text { stability }\end{array}$ & 3 & 1 & 2 & $1 / 2$ & $1 / 3$ & $1 / 2$ \\
\hline $\begin{array}{l}\text { Organization } \\
\text { management } \\
\text { structure }\end{array}$ & 2 & $1 / 2$ & 1 & $1 / 3$ & $1 / 4$ & $1 / 3$ \\
\hline $\begin{array}{l}\text { Value creation } \\
\text { ability }\end{array}$ & 4 & 2 & 3 & 1 & $1 / 2$ & 1 \\
\hline $\begin{array}{l}\text { Benefit and } \\
\text { risk sharing }\end{array}$ & 5 & 3 & 4 & 2 & 1 & 3 \\
\hline $\begin{array}{l}\text { Human } \\
\text { resources }\end{array}$ & 4 & 2 & 3 & 1 & $1 / 2$ & 1 \\
\hline Total & 19 & $53 / 6$ & $27 / 2$ & $61 / 12$ & $167 / 60$ & $61 / 12$ \\
\hline
\end{tabular}


Table 5 The weights of each index

\begin{tabular}{|l|l|l|l|l|l|l|l|}
\hline & $\begin{array}{l}\text { Strategic } \\
\text { concept }\end{array}$ & $\begin{array}{l}\text { Partner } \\
\text { stability }\end{array}$ & $\begin{array}{l}\text { Organization } \\
\text { management } \\
\text { structure }\end{array}$ & $\begin{array}{l}\text { Value } \\
\text { creation } \\
\text { ability }\end{array}$ & $\begin{array}{l}\text { Benefit } \\
\text { and } \\
\text { risk } \\
\text { sharing }\end{array}$ & $\begin{array}{l}\text { Human } \\
\text { resources }\end{array}$ & Weight \\
\hline $\begin{array}{l}\text { Strategic } \\
\text { concept }\end{array}$ & $1 / 19$ & $2 / 53$ & $1 / 27$ & $3 / 61$ & $12 / 167$ & $3 / 61$ & 0.050 \\
\hline Partner stability & $3 / 19$ & $6 / 53$ & $4 / 27$ & $6 / 61$ & $20 / 167$ & $6 / 61$ & 0.123 \\
\hline $\begin{array}{l}\text { Organization } \\
\text { management } \\
\text { structure }\end{array}$ & $2 / 19$ & $3 / 53$ & $2 / 27$ & $4 / 61$ & $15 / 17$ & $4 / 61$ & 0.076 \\
\hline $\begin{array}{l}\text { Value creation } \\
\text { ability }\end{array}$ & $4 / 19$ & $12 / 53$ & $2 / 9$ & $12 / 61$ & $30 / 167$ & $12 / 61$ & 0.205 \\
\hline $\begin{array}{l}\text { Benefit and risk } \\
\text { sharing }\end{array}$ & $5 / 19$ & $18 / 53$ & $8 / 27$ & $24 / 61$ & $60 / 167$ & $24 / 61$ & 0.341 \\
\hline $\begin{array}{l}\text { Human } \\
\text { resources }\end{array}$ & $4 / 19$ & $12 / 53$ & $2 / 9$ & $12 / 61$ & $30 / 167$ & $12 / 61$ & 0.205 \\
\hline Total & & & & & & & 1.000 \\
\hline
\end{tabular}

$$
A W=\left(\begin{array}{cccccc}
1 & 1 / 3 & 1 / 2 & 1 / 4 & 1 / 5 & 1 / 4 \\
3 & 1 & 2 & 1 / 2 & 1 / 3 & 1 / 2 \\
2 & 1 / 2 & 1 & 1 / 3 & 1 / 4 & 1 / 3 \\
4 & 2 & 3 & 1 & 1 / 2 & 1 \\
5 & 3 & 4 & 2 & 1 & 2 \\
4 & 2 & 3 & 1 & 1 / 2 & 1
\end{array}\right) \cdot\left(\begin{array}{c}
0.05 \\
0.123 \\
0.076 \\
0.205 \\
0.341 \\
0.205
\end{array}\right)=\left(\begin{array}{c}
0.300 \\
0.744 \\
0.459 \\
1.255 \\
2.084 \\
1.255
\end{array}\right)
$$

$\lambda=(0.3 / 0.05+0.744 / 0.1230+0.459 / 0.076+1.255 / 0.205+2.084 / 0.314+1.255 / 0.205) / 6=6.074$ $R I=1.26, C I=(\lambda-n) /(n-1)=0.015, C R=C I / R I=0.012<0.1$, complying with the consistency check.

The weight of each sub criterion index in the ability criterion was listed [3].The primary data are shown in table 6 and the normalized data is shown in table 7.

Table 6 The evaluation of raw data

\begin{tabular}{|l|l|l|c|c|}
\hline & $\begin{array}{l}\text { Core } \\
\text { competence }\end{array}$ & $\begin{array}{l}\text { Internal } \\
\text { coordination ability }\end{array}$ & $\begin{array}{l}\text { Operation } \\
\text { ability }\end{array}$ & $\begin{array}{l}\text { Emergency } \\
\text { response capability }\end{array}$ \\
\hline Core competence & 1 & 2 & 4 & 3 \\
\hline $\begin{array}{l}\text { Internal coordination } \\
\text { ability }\end{array}$ & $1 / 2$ & 1 & 3 & 2 \\
\hline Operation ability & $1 / 4$ & $1 / 3$ & 1 & $1 / 2$ \\
\hline $\begin{array}{l}\text { Emergency response } \\
\text { capability }\end{array}$ & $1 / 3$ & $1 / 2$ & 2 & 1 \\
\hline \multicolumn{1}{|c|}{ Total } & $25 / 12$ & $23 / 6$ & 10 & $13 / 2$ \\
\hline
\end{tabular}


Table 7 The normalized weights of each index

\begin{tabular}{|c|c|c|c|c|c|}
\hline & $\begin{array}{c}\text { Core } \\
\text { competence }\end{array}$ & $\begin{array}{c}\text { Internal } \\
\text { coordination } \\
\text { ability } \\
\end{array}$ & $\begin{array}{l}\text { Operation } \\
\text { ability }\end{array}$ & $\begin{array}{l}\text { Emergency } \\
\text { response } \\
\text { capability }\end{array}$ & Weight \\
\hline $\begin{array}{c}\text { Core } \\
\text { competence }\end{array}$ & $12 / 25$ & $12 / 23$ & $4 / 10$ & $6 / 13$ & 0.466 \\
\hline $\begin{array}{c}\text { Internal } \\
\text { coordination } \\
\text { ability } \\
\end{array}$ & $6 / 25$ & $6 / 23$ & $3 / 10$ & $4 / 13$ & 0.277 \\
\hline $\begin{array}{l}\text { Operation } \\
\text { ability }\end{array}$ & $3 / 25$ & $2 / 23$ & $1 / 10$ & $1 / 13$ & 0.096 \\
\hline $\begin{array}{c}\text { Emergency } \\
\text { response } \\
\text { Capability }\end{array}$ & $4 / 25$ & $3 / 23$ & $2 / 10$ & $2 / 13$ & 0.161 \\
\hline Total & & & & & 1.000 \\
\hline
\end{tabular}

$$
\begin{gathered}
A W=\left(\begin{array}{cccc}
1 & 2 & 4 & 3 \\
1 / 2 & 1 & 3 & 2 \\
1 / 4 & 1 / 3 & 1 & 1 / 2 \\
1 / 3 & 1 / 2 & 2 & 1
\end{array}\right) \cdot\left(\begin{array}{l}
0.460 \\
0.277 \\
0.096 \\
0.161
\end{array}\right)=\left(\begin{array}{c}
1.887 \\
1.120 \\
0.385 \\
0.647
\end{array}\right) \\
\lambda=(1.887 / 0.466+1.120 / 0.277+0.385 / 0.096+0.674 / 0.161) / 4=4.03, R I=0.89,
\end{gathered}
$$

$$
C I=(\lambda-n) /(n-1)=0.01, C R=C I / R I=0.011<0.1 \text {, complying with the consistency check. }
$$

The weights of sub criteria under the input criteria were listed. The primary data were shown in table 8 and the normalized data were shown in table 9.

Table 8 The evaluation of primary data

\begin{tabular}{|l|c|c|}
\hline & Asset scale & Advanced transport equipment \\
\hline Asset scale & 1 & 2 \\
\hline Advanced equipment & $1 / 2$ & 1 \\
\hline Total & $3 / 2$ & 3 \\
\hline
\end{tabular}

Table 9 The normalized weights of each index

\begin{tabular}{|l|c|c|c|}
\hline & Asset scale & Advanced transport equipment & Weight \\
\hline Asset scale & $2 / 3$ & $2 / 3$ & 0.667 \\
\hline Advanced equipment & $1 / 3$ & $1 / 3$ & 0.333 \\
\hline Total & & & 1.000 \\
\hline
\end{tabular}

$$
A W=\left(\begin{array}{cc}
1 & 2 \\
1 / 2 & 1
\end{array}\right) \cdot\left(\begin{array}{l}
0.667 \\
0.333
\end{array}\right)=\left(\begin{array}{l}
1.333 \\
0.667
\end{array}\right)
$$

$\lambda=(1.333 / 0.667+0.667 / 0.333) / 2=2, C I=(\lambda-n) /(n-1)=0, C R==0<0.1$, comply with the consistency check. 


\section{Strategic Partner Selection}

Absolute evaluation weight alternatives were obtained by using the formula of normalized weights (see table 10), 5 standards were used to valuating the system from 1-5 (representing very weak, weak, good, very good) [4]. The sorting job was done by expert scoring, then scores of the final four candidates as shown in Table 10.

Table 10 Candidate ratings

\begin{tabular}{|l|l|l|l|c|c|}
\hline Factor & Weight & $\begin{array}{l}\text { Shipping } \\
\text { enterprise1 }\end{array}$ & $\begin{array}{l}\text { Shipping } \\
\text { enterprise2 }\end{array}$ & $\begin{array}{l}\text { Shipping } \\
\text { enterprise3 }\end{array}$ & $\begin{array}{l}\text { Shipping } \\
\text { enterprise4 }\end{array}$ \\
\hline F1 & 0.032 & 2 & 2 & 3 & 2 \\
\hline F2 & 0.078 & 4 & 3 & 3 & 4 \\
\hline F3 & 0.048 & 4 & 3 & 2 & 3 \\
\hline F4 & 0.130 & 4 & 3 & 3 & 4 \\
\hline F5 & 0.216 & 3 & 4 & 4 & 3 \\
\hline F6 & 0.130 & 4 & 4 & 3 & 4 \\
\hline F7 & 0.122 & 3 & 2 & 3 & 3 \\
\hline F8 & 0.072 & 3 & 3 & 2 & 4 \\
\hline F9 & 0.025 & 2 & 3 & 3 & 3 \\
\hline F10 & 0.042 & 3 & 4 & 4 & 3 \\
\hline F11 & 0.071 & 4 & 3 & 3 & 3 \\
\hline F12 & 0.035 & 3 & 3 & 4 & 4 \\
\hline Total & & 3.403 & 3.237 & 3.176 & 3.416 \\
\hline
\end{tabular}

As seen from table 10, shipping enterprise 4 got the highest score by the comprehensive evaluation which is 3. 416. Theoretically, shipping enterprise 4 is the candidate.

\section{Summary}

In this paper, takes COSCO Group Co., Ltd as a case study, and makes a research on the combination of qualitative analysis and quantitative analysis. The using AHP model for the selection of shipping strategic partners is the main feature of the paper. In order to improve the competitiveness of shipping logistics and improve profits, the AHP method to select good partners is strongly recommended. It could make the overall functions of shipping logistics to minimize the risk, rationalize the operation and maximize the benefits.

\section{Acknowledgement}

This paper is subsidized by the innovation project of graduate education (2015YJ011) on Shandong Jiaotong University.

\section{References}

[1] Sui B. Cause of strategic technology alliance [J]. Scientific Management Research.2005, 23(02): 69-71. (In Chinese)

[2] Zhu G F. Decision making method for partner selection of strategic alliance [J]. Navigation of China, 2003 (01): 12-14. (In Chinese)

[3] Fan Y P, Chen J Y. Study on the decision making method of strategic alliance selection [J]. China Soft Science, 2000, (08): 102-105. (In Chinese)

[4] Chen J X, Fang J, He Z P. Optimization model of partner selection in logistics strategic alliance [J]. Statistics and Decision, 2010, (07): 167-169. (In Chinese) 